\title{
A 3-Dimensional Motion Sensing and Tracking System using Vector Analysis Method
}

\author{
${ }^{1}$ O.D. Alao, ${ }^{1}$ J.V. Joshua, ${ }^{1}$ A.O. Adebayo, ${ }^{2}$ G.A. Onanuga, ${ }^{1}$ M.A. Agbaje and ${ }^{1}$ I. Iyamu \\ ${ }^{1}$ Department of Computer Science and Information Technology, \\ School of Computing and Engineering Sciences, Babcock University Ilishan-Remo, Ikenne, Nigeria \\ ${ }^{2}$ Department of Computer Science, Ogun State College of Health Technology, Ilese, Nigeria \\ jimialao@hotmail.com
}

\begin{abstract}
The importance of security in today's world cannot be overemphasized. It is therefore, imperative to have a cheaper and lower resource requiring methods of achieving good security. Leaving home, work place, a child or somewhere important that needs supervision unattended is now a days not safe. There should be a form of supervision or surveillance either in person or by a machine. This research aimed at designing an electronic based device to provide such surveillance by sensing and tracking a single object in 3-Dimensional (3-D) motion within a defined space. Peering into the natural world it is found that animals such as dolphins, owls and bats locate objects and prey using sound, some animals even sense distortions in electromagnetic waves. Inspiration is not only garnered from nature but also from current technologies for this research. Three electronic sensors namely passive infra-red, active infrared and ultrasonic are evaluated and considered for designing a 3-D motion tracking system using mathematical method of vector analysis. An extensive look into the workings and features of all thethree sensors was done in order to find which sensor holds the greatest capacity for sensing an objects motion and obtaining the relative position of an object by the sensor in 3-dimensional space. Once achieved, the process of measurement can be done repeatedly at distinct time intervals to track the motion of the object in 3D space. The major benefit of this research is for the security and surveillance of property or personnel but is not limited to this. There is also an opportunity for applications in other areas such as in sports for tracking the 3D motion of an athlete and can also assist in evaluating player statistics such as speed and acceleration.
\end{abstract}

Key words: Sensors, motion tracking, surveillance and vector analysis, supervision, evaluating player, opportunity

\section{INTRODUCTION}

A major problem in security and surveillance is sensing movement and tracking of such movement in restricted areas. Motion sensors are devices that turn other equipment on or off in response to the presence (or absence) of people in a defined area. However, motion sensors have become widely used in past years as the device and has become more relevant in automation and security systems. A motion sensor senses motion and determines the occupancy status of an area. The earliest recorded sensing technologies involved tribes which would tie object together and place them strategically on pathways and bushes on the perimeter of living or farming areas. This would produce loud clanking and other sounds when the rope/string was tugged on that would be easily audible and thus, alert them to a possible intrusion. The sounds also acted as a deterrent to hopefully scare away persons or predators.

In the late 1800 , the first motion detection system radar was pioneered by Heinrich hertz. He studied the properties of waves and found that waves could bounce off objects and had different speeds of dispersion. Though motion detection became a largely used phenomena during World War II because of the need to track air and naval vessels and due to the advances in study of the properties of waves and wave dispersion. The use of radar lead to other uses for motion detectors after World War II. The first motion detector which was a burglar alarm was invented in the 1950s by Samuel Bango.

Corresponding Author: O.D. Alao, Department of Computer Science and Information Technology, School of Computing and Engineering Sciences, Babcock University Ilishan-Remo, Ikene, Nigeria, jimialao@hotmail.com 
He applied the fundamentals of radar to ultrasonic waves (Anonymous, 2019a). Ultrasonic waves are on a frequency that the normal human ear cannot pick up hence Samuel Bango's motion detector made use of Doppler's effect to figure out the difference between the frequencies of waves of a moving object (Anonymous, 2019b). Down the line motion sensors began to make use of multiple technologies to detect movement and currently most of the motion sensors work on the principle of Samuel Bango's detector.

This study started with a discussion on the comparison of the functionality and features of the different types of proximity sensors chosen for the study followed by a description of the design, implementation and testing of the motion sensing device. The study was concluded by examining the limitations of the sensors, possible solutions to the limitations and with a recommendation for further study.

Problem statement: Human beings have a natural desire to protect things of values such as their loved ones to property (objects and resources) from external interference which may lead to loss, harm or destruction. The problem of securing these valuables is that a person has limited range of perception, that is a single person can only watch over that which is in their immediate vicinity, in many cases leaving vast areas unattended. The security and protection of these valuables may require different approaches but from time immemorial the most basic method is to watch over things ourselves. In other words to personally surveilthe object or entity and the space surrounding it. Since, it is not possible to be in two or more places at once. Surveillance methods based on sensors is the technological solution to this quandary and is preferred in many cases because it eliminates the need for added human resources (e.g., a bunch of security guards).

Objectives of the study: To carry out a review of the workings and features of passive infra-red, active infrared and ultrasonic sensors in order to find which sensor holds the greatest capacity for sensing objects motion. To design a sensor based 3-D motion tracking system using vector analysis method with motion of the entity displayed in real-time. To implement and test device design in two above.

Significance of the study: Today, motion sensors can be found as part of many industrial and workplace security systems. This device has the added feature of tracking the object's (/intruder's) motion, thus, providing more detailed information without the sometimes extravagant cost of video surveillance systems. In the cases of human intrusion both infrared and ultrasonic sensors have the added advantage of operating without a light source.

Motion detection: Motion can be detected by measuring change in speed or vector of an object or objects in the field of view, this can be achieved either by mechanical devices that physically interact with the field or by electronic devices that quantify and measure changes in the given environment. Motion can be detected by sound (acoustic sensors), opacity (optical and infrared sensors, video image processors), geomagnetism (magnetic sensors, magnetometer) reflection of transmitted energy (infrared laser radar, ultrasonic sensors and microwave radar sensors), electromagnetic induction (inductive-loop detectors) and by vibrations.

Motion detector: A motion detector is an electronic device that detects the physical movement of a given area and transmits the movement into a digital signal. The motion detector may be electrically connected to devices such as security lighting, sound alarm and the likes. Motion detection sensors are use in a large variety of applications and as a result many different types of these sensors are available. Motion detectors are mainly used for security system. For example, motion sensors are typically positioned near doors and windows for monitoring the area around a building. Upon detecting motion, they generate an electrical signal that is transmitted to a pre-selected audible alarm or lighting device which is then activated (Anonymous, 2019c, d).

Types of sensors used for motion detectoin: Motion detectors spot the movement in an areas covered by the security sensors. It transmits high frequency radio wave signals and gives a warning that someone is entering into sensor area. Motion detection sensors can be broadly classified as follows:

Gesture sensors: Which make use of infrared lighting elements and digital screens to detect hand motions and gestures with the aid of learning algorithms.

Vibration motion sensors: This sensors use the piezoelectric effect or the ability of objects to create an electric field to detect motion.

Video motion sensors: This combines video cameras with advanced signal processing. There are a wide variety of motion detectors available currently, for a better understanding of motion detectors sensors, the next section provides a detailed description of the different types of sensors used for motion detection. 

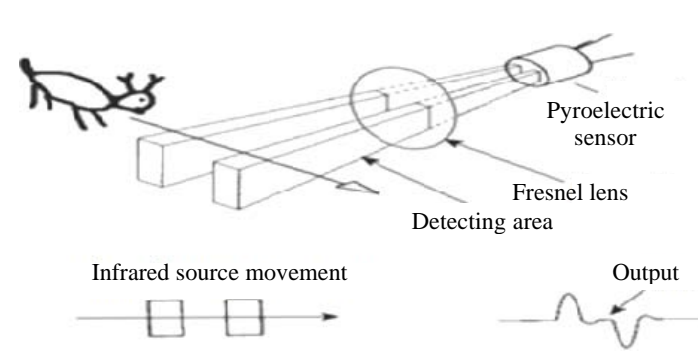

Fig. 1: Passive infrared sensor operation

Infrared sensors: Infrared (IR) sensors are widely known in the act of intrusion detection and in fire or smoke detection. These sensors have basically two forms: active and passive. Active IR sensors has a radiation source and an IR sensor which is sensitive to interruptions in the radiation sensed from the source. These sensors are used for intrusion detection by providing a path of radiation from the source to the sensor in a place where the path is likely to be interrupted by an intruder. A passive infrared motion detection system detects heat energy radiated or emitted by an object such as a body of a person moving across a field of view of a heat sensor (Anonymous, 2019d). Passive infrared motion detectors generally use an optical collection system and multiple sensing elements of alternating polarity to create a detection pattern in the volume of interest (Agarwal, 2017). Passive IR detectors also employ a group of radiation sensors coupled through amplifiers to a logic circuits. The radiation detector sensors detect changes in ambient infrared radiation. The detection system has an electrical circuit operatively coupled to the heat sensors for producing a detection signal in response to the heat sensor detecting a change in temperature caused by the body heat of a person entering the detection pattern as shown in Fig. 1. Passive infrared motion detection and occupancy sensors employ array of Fresnel lenses covering an entrance aperture (Anonymous, 2019a). This lens array is illuminated by thermal infrared radiation from the object of interest.

Infrared radiation theory: Infrared waves are not visible to the human eye in the electromagnetic spectrum infrared radiation can be found between the visible and microwave regions, the infrared waves typically have wavelengths between 0.75 and $1000 \mu \mathrm{m}$.

The infrared spectrum can be split into near IR, mid IR and far IR. The wavelength from $0.75-3 \mu \mathrm{m}$ is known as the near infrared region. The region between 3 and $6 \mu \mathrm{m}$ is known as the mid infrared region and the infrared region which has a wavelength higher than $6 \mu \mathrm{m}$ is known as far infrared.
Foundations of infrared sensors: nfrared sensors and their theory have been around since the 1800 when F.W Herschel discovered infrared light (Agarwal, 2017). Herschel conducted an experiment using a prism to reflect light from the Sun and was able to detect the presences of infrared radiation beyond the red part of the visible spectrum using a thermometer to measure an increase in temperature.

Working principle of infrared sensors: The physics behind infrared sensors is governed by three laws:

Planck's radiation law: Every object at a temperature $\mathrm{T} \bullet 0$ $\mathrm{K}$ emits radiation.

Stephan boltzmann law: The total energy emitted at all wavelengths by a black body is related to the absolute temperature:

$$
\mathrm{W}_{\mathrm{b}}=\mathrm{s} \mathrm{T}^{4}
$$

Where:

$\mathrm{W}_{\mathrm{b}}=$ Ttotal energy emitted

$\mathrm{s}=$ Constant $=5.67 \times 10^{-8} \mathrm{~m}^{-2} \mathrm{~K}^{-4}$

$\mathrm{T}=$ Temperature of the object

Wein's displacement law: Objects of different temperature emit spectra that peak at different wavelengths. All objects which have a temperature greater than absolute zero $(0 \mathrm{~K})$ possess thermal energy and are sources of infrared radiation.

Radar sensors: Radar based motion sensors emit a continuous-wave microwave signal that produce a heat frequency that is proportional to range. Such microwave sensors for detecting the presence of moving objects and direction of movement are found in a variety of applications such as door openers, security systems and toilet flushers.

Such detector typically comprise of a higher radio frequency circuit comprising an oscillator for generating a high frequency oscillating signal an antenna or antennas for transmitting and receiving oscillating signals and a mixer for modulating oscillator signals with the received signal. In other to determine the direction of motion of an object the oscillating signal is mixed with the receive signal to generate two output Intermediate Frequency (IF), the combination of which determines the direction of movement of an object towards or away from the sensor.

Ultrasonic sensors: Ultrasonic motion sensors transmit and receive ultrasonic sound in a region of interest and when an object in a range of ultrasonic motion sensor is 


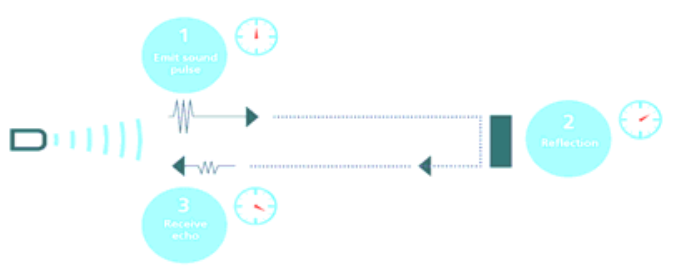

Fig. 2: Ultrasonic sensor operation

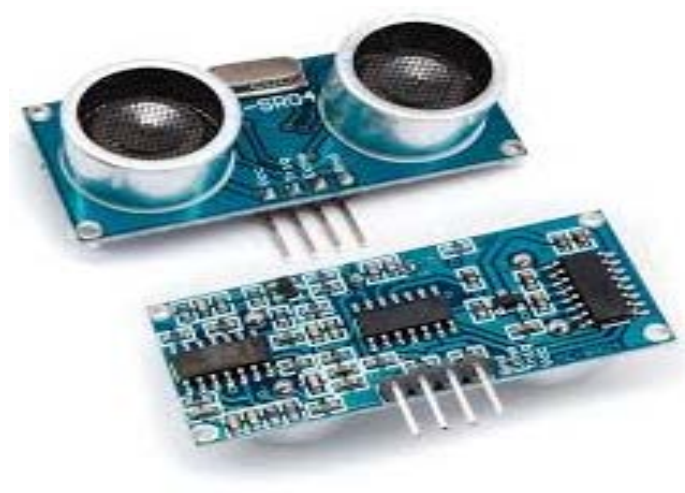

Fig. 3: HC-SR04 ultrasonic sensor

detected and an alarm signal is produced. The effective range of an ultrasonic motions detectors differ from design range. The energy most times comes from a quartz crystal transducers and when the sound waves bounce off a moving object or object in motion, the sensor measures the displacement or reflection off the moving object. It is mostly used with automated and sliding doors but the down side is that it can be easily blocked or disrupted.

Operation of the ultrasonic sensors: When an electrical pulse of high voltage is applied to the ultrasonic transducer it vibrates across a specific spectrum of frequencies and generates a burst of sound waves as shown in Fig. 2. Whenever any obstacle comes ahead of the ultrasonic sensor the sound waves will reflect back in the form of echo and generates an electric pulse (Zungeru, 2013). It calculates the time taken between sending sound waves and received echo. The echo patterns will be compared with the patterns of sound waves to determine detected signal's condition.

Description of the ultrasonic sensor: The HC-SR04 ultrasonic sensor shown in Fig. 3 uses sonar to determine distance to an object like bats do. It offers excellent non-contact range detection with high accuracy and stable readings in an easy-to-use package. The transmitter

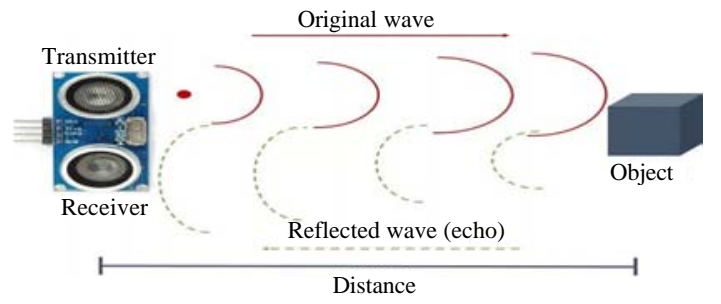

Fig. 4: Ultrasonic sensor distance operation

pin sends a signal: a high-frequency sound. When the signal finds an object, it is reflected and the transmitter (echo pin) receives it shown Fig. 4.

The time between the transmission and reception of the signal allows us to obtain the full distance travelled by the ultrasonic pulse wave. This is possible because the velocity of sound in the air is $340 \mathrm{~m} / \mathrm{sec}$ and from the distance formula:

$$
\text { Distance travelled }=(\text { Velocity }) \times(\text { Time })
$$

Since, the ultrasonic pulse wave travelled to and from the object the distance to the object is obtained by dividing the distance travelled by two:

Object distance $=($ Distance travelled $) /$ two

All these calculations are done on sensor circuit and the final distance is the output.

Microwave motion sensors: The microwave sensors generate microwave pulses in a particular field or area and then calculate their reflection from an objects in the area. They cover wider areas than the infrared sensors. Microwave sensors are very sensitive and are sometimes seen on in non-metallic objects. Microwave sensors continually emits microwaves and gets triggered when a body reflects the waves back.

Tomographic motion sensor: This sensors generate radio waves and detect when the radio waves generated are troubled they are often used in a way that generates a radio wave mesh that covers wide areas. They have the ability to detect through walls and other obstructions. They are mostly used in ware houses and storage units and they are expensive.

Hybrid motion sensors: This sensors combine both the active and passive sensors into one sensor it activates an alarm or notifies user only when motion is picked up by both sensors an example is the hybrid sensor is the passive infrared and the microwave sensor. 
Table 1: Comparison of ultrasonic and infrared sensors

\begin{tabular}{|c|c|c|}
\hline Features & Ultrasonic sensors & Infrared sensors \\
\hline Detection range & $\begin{array}{l}\text { Suitable to detect objects which are more than } 1 \mathrm{~m} \text { away } \\
\text { Capable to place objects within } 5 \mathrm{~mm} \text { more accurately } \\
\text { Some ultrasonic sensors detect objects with max. Range of } 20 \mathrm{~m} \\
\text { The object detection depends on shape, size and orientation }\end{array}$ & $\begin{array}{l}\text { More appropriate for targets } \\
\text { which are closer than } 10 \mathrm{~mm}\end{array}$ \\
\hline Ability to measure distance to soft objects & Not easily affected & Easily affected \\
\hline $\begin{array}{l}\text { Interference from light sources } \\
\text { (i.e., sunlight, fluorescent bulbs, etc.) }\end{array}$ & Unaffected & Affected \\
\hline Frequency range & Ultrasonic/ultrasound devices operate from $20 \mathrm{kHz}$ up to several $\mathrm{GHz}$ & $430 \mathrm{THz}$ down to $300 \mathrm{GHz}$ \\
\hline Wavelength & Ultrasonic waves use wavelengths of about $1.9 \mathrm{~cm}$ or less & $700-1 \mathrm{~mm}$ \\
\hline
\end{tabular}

Applications areas of motion sensors: The main purpose of a motion detector is to detect or notice moving objects, mainly people but it has been known to be applied in the following ways:

Intruder alarms: it is mainly used in buildings such as residential or government properties to keep unauthorized individuals out of the premises and to check when unauthorized personnel strays into a restricted area.

Ticket gates: Motion sensors are used at automatic and sometimes unmanned ticket gates to signify when the vehicles come through, so as to know when to open and when to close.

Automatic lights: Motion sensor are used in making automatic lights now a days because it senses when someone steps into the room or building and based on that it turns on the light.

Water faucets and toilets: Motion sensors are used in water faucets around the world. Now a days in toilets people don't need to turn on/off the water faucet anymore, they can just put their hand under the faucet once the motion sensor senses the heat emitted from the hand it opens the faucet and water start flowing and when done, it turns off. It is the same for toilets hand dryers.

\section{Ultrasonic versus infrared sensors}

Review of related works on motion sensing and tracking systems: Xu et al. (2019) proposed a multi-sensor hybrid motion tracking method, they viewed human motion tracking as a multi-target tracking problem towards numerous body joints. Inertial-measurement-unit-based human motion tracking technique stands out and has been widely used in body area network applications. However, it has been facing the tough problem of accumulative errors and drift. In this study, they propose a multi-sensor hybrid method to solve this problem. Simulation results show that the proposed method in this paper has both spatial and temporal advantages, compared with traditional sole inertial or time-of arrival-based tracking methods. Furthermore, proposed method is verified in 3D practical application scenarios. Compared with state-of-the-art algorithms, proposed fusion method shows better consistency and higher tracking accuracy, especially, when moving direction changes (Table 1).

Sun et al. (2017) proposed a method for detecting the motion of human lower limbs including all Degrees of Freedom (DOFs) with the use of inertial sensors which permits analyzing the patient's motion ability. This method is applicable to arbitrary walking directions and tracks of persons under study and its results are unbiased as compared to therapist qualitative estimations. Using the simplified mathematical model of a human body, the rotation angles for each lower limb joint are calculated from the input signals acquired by the inertial sensors. Finally, the rotation angle versus joint displacement curves are constructed and the estimated values of joint motion angle and motion ability are obtained. The experimental verification of the proposed motion detection and analysis method was performed which proved that it can efficiently detect the differences between motion behaviors of disabled and healthy persons and provide a reliable quantitative evaluation of the rehabilitation state.

Pavithra et al. came up with an automated video surveillance system for real time observation of people in a busy environment. The system when realizes human entry, it is processed in a second and an alert is produced.

Mohammadzadeh et al. (2015) developed and evaluated the feasibility of a wearable, sensor-based motion tracking system that provides an economical and quantitative means of recording upper limb motion for physical rehabilitation. The tracking system is comprised of a wirelessly connected network of Inertial Measurement Units (IMUs), each containing a gyroscope and an accelerometer. Two IMUs were rigidly attached to each subject's forearm and upper arm. A trajectorizing algorithm was developed to estimate the three dimensional upper limb motion based on the measurements of the IMUs. A major advantage of the algorithm is that it allows the IMUs to be attached with arbitrary orientation to each limb and no manual 
anthropomorphic measurements need to be performed. By recording specific, known motions, the sensors can be calibrated with respect to their orientation in space and with respect to their orientation relative to their respective body segments. Their results showed that an IMU-based motion tracking system can be realistically used to accurately track a patient's motion without the need of numerous sensors or an overly complicated set-up.

Chodon et al. in their study developed a Passive Infra-Red (PIR) sensor based security system which saves power consumption and memory of the recording system. When an intruder comes in the detection range of the PIR sensor it actuates a lightning system and a webcam. It will start to record and save the video. Once the intruder goes out of range of the sensor, the webcam and the lighting system get turn off thereby saving power and memory space.

Ching et al. developed a monitoring system is that utilizes a motion detection technology with modified internationally recognized algorithms, implemented in $\mathrm{C}$ sharp and MATLAB programming language. The result of this study was beneficial and assisted users on effective motion detection and analysis. Four different motion detectors were being compared. In conclusion, an effective motion assessment and monitoring system was developed for the improvement of the motion detection ability.

Li et al. (2009) developed a baby monitor that integrates a low-power doppler radar that can detect minute movement induced by breathing. If no movement is detected within $20 \mathrm{sec}$, an alarm goes off. These types of sensors are used in many other systems that deal with tracking of objects ranging from humans, to ships, to pipes, explosives etc. and applications like search and rescue missions, pipe inspection and offshore wind turbines.

\section{MATERIALS AND METHODS}

The methodology used in this research is procedural; research, experimentation, testing and then implementation. Initial broad spectrum research was done into security and surveillance systems, sensory devices and motion tracking technologies. From this device and system components were selected after a more fine-tuned and detailed review of the current technologies was done. Also system requirements were developed.

Three sensors where selected for consideration, namely; passive infrared, active infrared proximity sensors and ultrasonic proximity sensors. During experimentation, the infrared (active) and the ultrasonic sensors were compared on critical sensing criteria such as range,

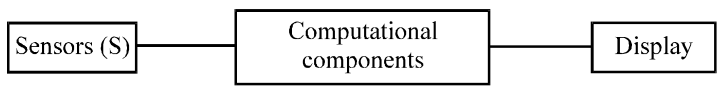

Fig. 5: Basic design

sensitivity and firing intervals to determine sensor selection. Once the sensor was selected, the basic system design was developed and tested in phases. To track the 3D motion of the object, a mapping method (triangulation) was adopted to make use of the continuous distance measurements obtained by the sensors. This mapping technique was applied using Software (MATLAB), thus, resulting in a real-time display of the objects motion in $3 \mathrm{D}$.

Basic design: When bats, dolphins or other animals are trying to locate objects, they release pulses of sound waves which when reflected by objects returns to them. How long this takes tells the animal how far away the object is. This is the working principle behind sonar and radar systems. An application of this principle was used to develop the proposed system. The system consist of three major parts namely, the sensors, the computational components and the display unitas shown in Fig. 5.

Motion tracking in 3D: To track an object in 3D using distance readings (which are essentially, 1 dimensional straight lines) a minimum of three readings are required. This means that the system must have at least three active sensors. Testing was be done with 3 or more sensors in order to determine the most effective setup. These distance readings obtained from the sensors were analyzed using vector analysis method to calculate the relative position of the object in three dimensional space. It may also be done using polar co-ordinate. The analysis was carried out and then written into the system control code in MATLAB (Appendix).

Computational components: The information obtained from the sensors must be processed in order to obtain a distance reading. In order to achieve this because there exists in the market multiple ultrasonic sensors which come integratedon a mini-board where distance readings are computed electronically. To get these readings it was necessary to come up with a pulsing sequence for the sensors and in order to do this they needed to be controlled. This can be done by programming a microcontroller for the sensors and other peripheral devices. Multiple microcontrollers where compared on criteria such as number of input/read pins and the Arduino Uno was selected. By applying an algorithm 


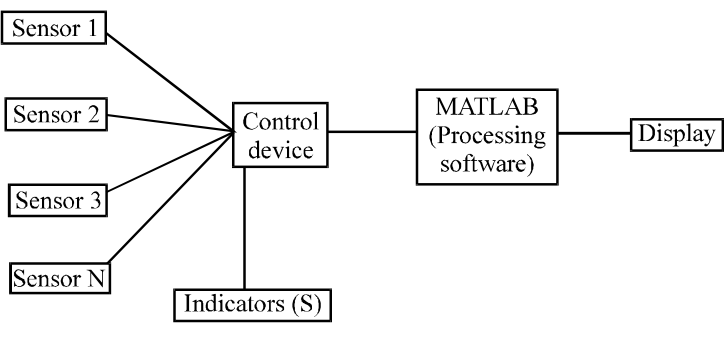

Fig. 6: Initial design of the system

code to the readings obtained, graphing/mapping software was then used to display the coordinates obtained from the algorithm in 3D. The software used was MATLAB and was selected primarily because it can be directly linked to the microcontroller thus eliminating the need for the interim storage of sensor data in files or a database, thereby reducing latency. The use of MATLAB and the Arduino microcontroller required a computer. The integration of all the above mentioned aspects of the design led to the primary system expressed in Fig. 6.

Method of sensor selection: The appropriate sensor type used for the motion detection and tracking system was chosen by evaluation of different sensor types. The infrared sensor types: passive infrared sensor and active infrared sensor was reviewed, also reviewed was the ultrasonic sensor, the tomographic sensor, the microwave sensor and the hybrid sensors.

The availability of some of the sensors were questionable as some of them are difficult to acquire in this part of the world (Africa) and the cost of the sensor is also a major factor in the sense that some of the sensors, although, efficient are not really easy to get due to their cost, the infrared sensor, although, efficient is very expensive than the other sensors reviewed.

The ultrasonic sensor has the highest and widest signal detection range of all the sensors chosen for this study, the signal detection range of the ultrasonic sensor is about $1 \mathrm{~m}$ for accurate detection but the infrared sensor has a detection range of $10 \mathrm{~mm}$.

Another evaluation criteriais the climate and weather condition in this part of the world (Africa), since, infrared sensors have difficulties with detecting more than one object with similar heat signatures that is a significant problem with the infrared and the pyro electric sensor but it isn't a problem with the microwave sensor and the ultrasonic sensor.

Compatibility between the graphing software and microcontroller was also put into consideration for the purpose of this study. The graphing software used is MATLAB and it has been seen that the ultrasonic sensor chosen is very compatible with it and with the Arduino microcontroller used. Finally, ultrasonic sensors when compared to infrared sensors have the clear advantage of little interference due to excess light or heat sources being present and the sensors works perfectly in the dark.

Method of selection of the microprocessor and interface software: The microprocessor chosen for this study was the Arduino Uno and this was chosen through the evaluation of different microprocessors ranging from the Arduino Uno, Arduino Mega, Arduino Leonardo, Raspberry $\mathrm{Pi}$, Beagle bone amongst others. The microprocessors were evaluated based on:

Form factor: The form factor of the Arduino Uno microprocessor is the smallest and the most portable of all the microprocessors the Arduino Mega, according to its name has a significantly big form factor same with the beagle bone and Arduino Leonardo.

Number of pins: the Arduino Uno has fourteen digital Onput/Output $(\mathrm{I} / \mathrm{O})$ pins and six analog pins which for its form factor is a very impressive microprocessor.

Onboard USB controller: The Arduino Uno microprocessor is one of the microprocessors that has an onboard USB controller making it able to connect the board to the computer via. USB cable which does a dual purpose of supplying power and acting as a serial port to interface the Arduino and the computer.

Method of selection of alerting motion of an entity within the sensing range: An alert is a sound, warning or anything that catches the attentions of a person, indicating that some predefined events has occurred. For the purpose of this research, both the beeper and the LED was used. The method used to make this decision was comparison of both components with the limitation of hearing for people with hearing disability and to increase the chances or probability of getting the attention of the person being supervised or monitored.

Method of selection of the display in real-time: Another objective of the motion tracking deviceis to display the 3D motion of the entity in real-time". There are so, many software that can be used to display the output of this research in 3D. One of such software is the Flexpro. Flexpro is a software application for analyzing and presenting data. All data, analyses and presentations are stored in an object database. The structure of the database is similar to that of a file system on a hard drive. IGOR Pro is another software which is primarily known for 
its graphic capabilities and is often used to generate plots for scientific and other publications. Another software is the MATLAB which is the easiest and most productive software environment for engineers and scientists. MATLAB is alsoused primarily for numeric computing. Scilab is another software that is free and open-sourced, cross platform numerical computational package and a high-level, numerically oriented programming language. It can be used for signal processing, statistical analysis, image enhancement, fluid dynamics simulation, numerical optimization and modeling, simulation of explicit and implicit dynamical system. In essence, it has better visualization of plots and simulations than other graphing software.

The method of selecting a 3D output software is by comparing the pros and cons of each software. For this research, MATLAB was chosen, the reason being that after all the comparison was done, MATLAB's graphical output is optimized for interaction, data entry can be plotted easily and modification of colors, size scales etc can be done with ease. It also includes high-level commands for two-dimensional and three-dimensional data visualization, image processing, and presentation graphics. It also includes low-level commands that allow you to fully customize the appearance of graphics as well as to build complete graphical user interfaces on the MATLAB applications.

Implementation overview: The 3D motion sensing and tracking system was implemented in phases based upon major areas of functionality namely, hardware and software. The system is meant to accomplish two things. Alert users of motion within the 3D sensing region and track motion within the region in 3 dimensions of space. The first was accomplished by setting the system to turn on the LED and/or buzzer when an echo signal is detected by the sensor. Once an object is detected within sensing range a firing sequence is initiated. Each sensor is triggered to release pulses in a continuing sequential order. The purpose of the sequence is to reduce the chance of error in the distance readings due to interference from other sensor pulses. The delay between pulse triggers must be enough for the triggered sensor to detect the associated echo. This delay is a function of the maximum distance to be measured by each sensor. The sensor uses the return time of the pulse to determine the distance to the object it bounced off. These values are fed into the graphing software for each round of the sequence for which a point is plotted in 3 dimensions. Figure 7 below shows a diagrammatic representation of the entire system.

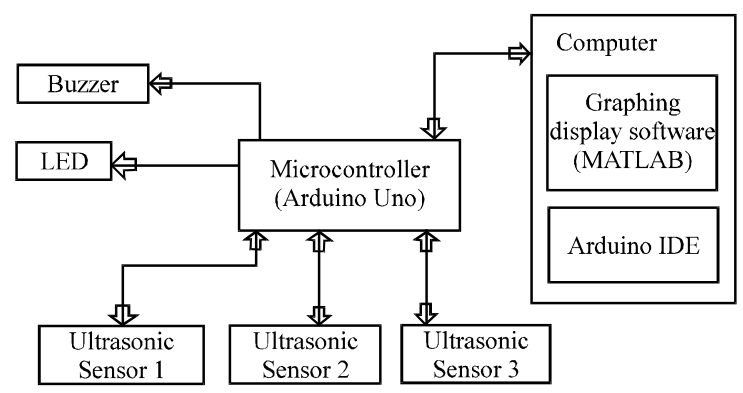

Fig. 7: Block diagram of 3D motion sensing and tracking system

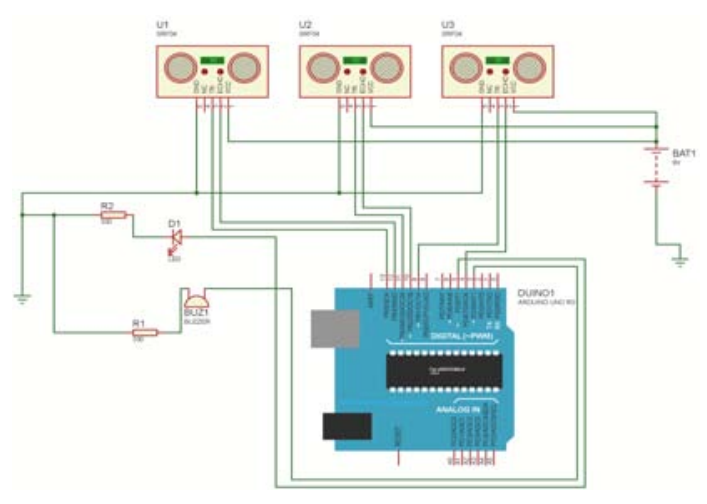

Fig. 8: Circuit diagram of 3D motion sensing and tracking system

Phase one; hardware connection: The hardware system components were connected as shown in the circuit diagram above in Fig. 8. The trigger and echo pins of the ultrasonic sensor are connected to the Arduino Uno digital input ports. The power supply voltage (VCC) and ground were also connected to the corresponding pins on Arduino board. The fourth sensor is to be added to spare pins of the microcontroller. The LED and Buzzer are connected to separate digital input ports in series with a 330 resistor and the microcontroller connected via. the USB to the computer.

Phase two; sensor setup and positioning: Positioning is important because the three sensors must be placed at the be placed at a level height from the ground with the exception starting co-ordinates of the $3 \mathrm{D}$ vectors used to compute the position of the object. Two ultrasonic sensors must also of one sensor which is placed higher. The sensors must not be placed within the sensing range of all other sensors and also not be placed next to one another or side by side. If the sensors are placed at the same height two of the sensors should be placed orthogonal to the third facing each other (Fig. 9a, b). The 

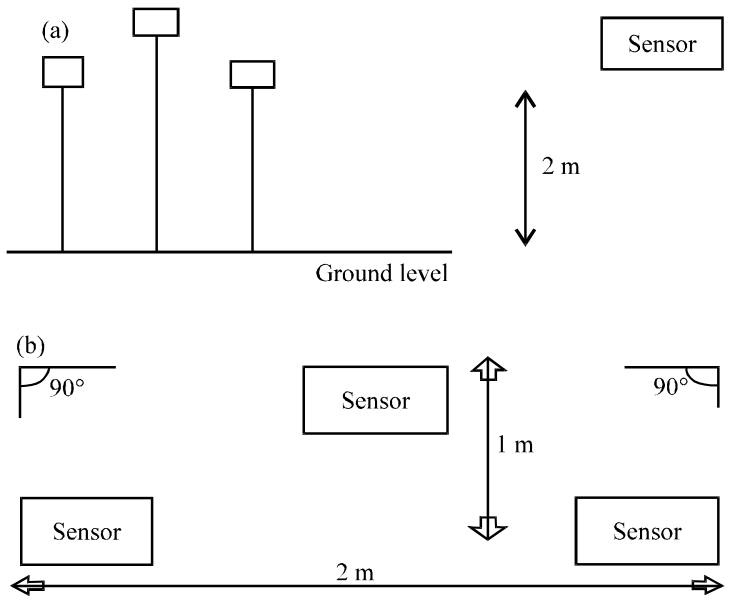

Fig. 9: a) Level view of sensor positioning and b) Aerial view of sensor positioning

third sensor may be place anywhere within $1.5 \mathrm{~m}$ from the center of the other sensors but placed higher by a minimum of $0.5 \mathrm{~m}$.

Phase three; software setup: It is critical that the Arduino and HC-SR04 sensor add-ons are correctly downloaded into MATLAB, also the HC-SR04 file must be added to the Arduino library (instruction for this can be found in MATLAB add-ons or math works website). Necessary adjustments to the sensor libraries. Cppcode file are needed to limit the sensors echo delay time by doing so, the effective distance is limited, so as to keep all sensors are out of range of the rest. The microcontroller is programmed for control in MATLAB programming language via. the graphing Software (MATLAB). The code implements an algorithm which dictates when each sensor is to release a pulse and how long it may wait for a response (the firing sequence). The code also implements the control variants for when the LED or Buzzer should trip on then off as an alert to motion with respect to the HC-SR04 sensor. The code also sets the output frequency ensuring it is in the ultrasonic range. The code on the Integrated Development Environment (IDE) also serializes the distance readings and passes them on to the graphing software in real-time. Finally, the system hardware is controlled via. the graphing software, this necessitates the download of the necessary support packages to make the link to the Arduino board.

Testing: Different forms of testing were done at all three phases. This testing strategy is described below.

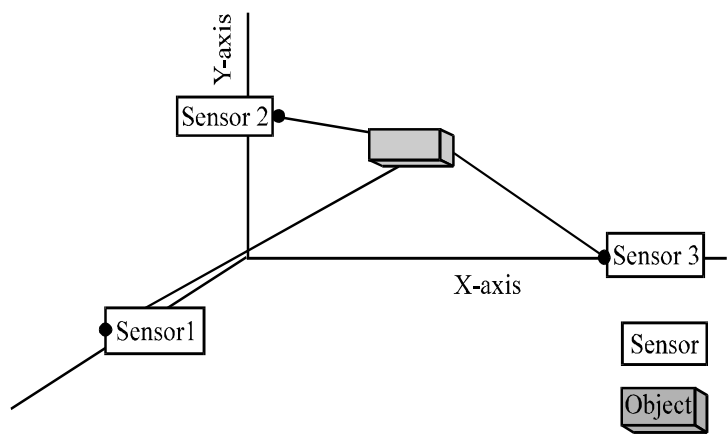

Fig. 10: Instant measurement/reading from the actual sensor position

Unit testing: The system has been tested to ensure that it is error-free. In each phase, each part of the system were tested separately as an addition to the system setup at that point of completion:

- At the completion of phase 1 a multi meter is used to test all physical connections made by the hardware components

- After phase 2 completion, distance measurements between sensors were taken.

- At the completion of phase 3, test code for the LED, Buzzer and sonsors was ran in the Arduino IDE to ensure functionality

- Testing was also done in MATLAB to ensure the connection between MATLAB, Arduino board and the sensors (Appendix for all testing code)

Complete system testing: At this stage, the system is tested as a whole. The essence of this testing is to ensure the system works as intended. This testing brings to light any adjustments to be made in order to meet the prior stated requirements.

Vector analysis method for motion tracking in 3D using: Let each distance reading from the sensors 1-3 to the object be scalar representation of the vectors SZ, SY and SX, respectively as shown in Fig. 10. The origin of all the above mentioned vectors is determined by the relative position of the placements during setup (note that they are all along some point of each axis). For the sake of simplicity all these origins are on the positive side of their axis. Knowing that the vectors converge at the object this gives us a point $\mathrm{P}(\mathrm{x}, \mathrm{y}, \mathrm{z})$ at which the vectors $\mathrm{SZ}, \mathrm{SY}$ and SX all end. Consider the equation below which represents magnitude of a vector $\mathrm{AB}$ :

$$
\text { Distan ce } \mathrm{AB}=\sqrt{\left(\mathrm{x}_{2}-\mathrm{x}_{1}\right)^{2}+\left(\mathrm{y}_{2}-\mathrm{y}_{1}\right)^{2}+\left(\mathrm{z}_{2}-\mathrm{z}_{1}\right)^{2}}
$$


where, $x_{1}, y_{1}$ and $z_{1}$ are the start point coordinate for the vectors and $x_{2}, y_{2}$ and $z_{2}$ are the end point co-ordinate for the vector. The vectors SZ, SY and SX are the shared converging point coordinate (this is the coordinate to be calculated).

Mathematical solution to find the convergence point of three vectors in $3 \mathrm{D}$ space

Lemma: Given a finite set of fixed points $Q=\left\{\mathrm{S}_{1}, \mathrm{~S}_{2}, \mathrm{~S}_{3}\right\}$ which exist in the set $/ R^{3}$. Let $P=(x, y, z)$ be a point in space. Then the norm $\left\|\mathrm{S}_{\mathrm{i}}-\mathrm{x}\right\|(\mathrm{I}=1,2,3)$ determines $\mathrm{P}$ uniquely. Where, $S_{1}=(a, 0,0), S_{2}=(0, b, 0), S_{3}=(0,0, c)$. Here, a-c are positive real numbers.

Method approach: Using the magnitude equation of three converging vectors to find the co-ordinate of the point of convergence $\mathrm{P}=(\mathrm{x}, \mathrm{y}, \mathrm{z})$.

\section{Known values:}

- Origin point of all three vectors $\mathrm{S}_{1}, \mathrm{~S}_{2}, \mathrm{~S}_{3}$

- Magnitude (length) of all three vectors

The magnitude formula for 3D vector is given by: For a vector $\mathrm{A}$ in $3 \mathrm{D}$ space with axis $\mathrm{X}-\mathrm{Z}$ its magnitude is:

$$
|A|=\sqrt{\left(\left(\mathrm{x}_{2}-\mathrm{x}_{1}\right)^{2}+\left(\mathrm{y}_{2}-\mathrm{y}_{1}\right)^{2}+\left(\mathrm{z}_{2}-\mathrm{z}_{1}\right)^{2}\right)}
$$

where, the co-ordinate $\left(\mathrm{x}_{1}, \mathrm{y}_{1}, \mathrm{z}_{1}\right)$ and $\left(\mathrm{x}_{2}, \mathrm{y}_{2}, \mathrm{z}_{2}\right)$ are co-ordinates of the start point and end point of the vector, respectively. Hence, $\left(\left(\mathrm{x}_{2}-\mathrm{x}_{1}\right),\left(\mathrm{y}_{2}-\mathrm{y}_{1}\right)\right.$ and $\left(\mathrm{z}_{2}-\mathrm{z}_{1}\right)$ in $(1)$ are the $\mathrm{i}, \mathrm{j}, \mathrm{k}$ components of vector $\mathrm{A}$.

Similarly given three vectors A-C as shown in Fig. 11 with lengths $\mathrm{A}-\mathrm{C}$ and corresponding starting points

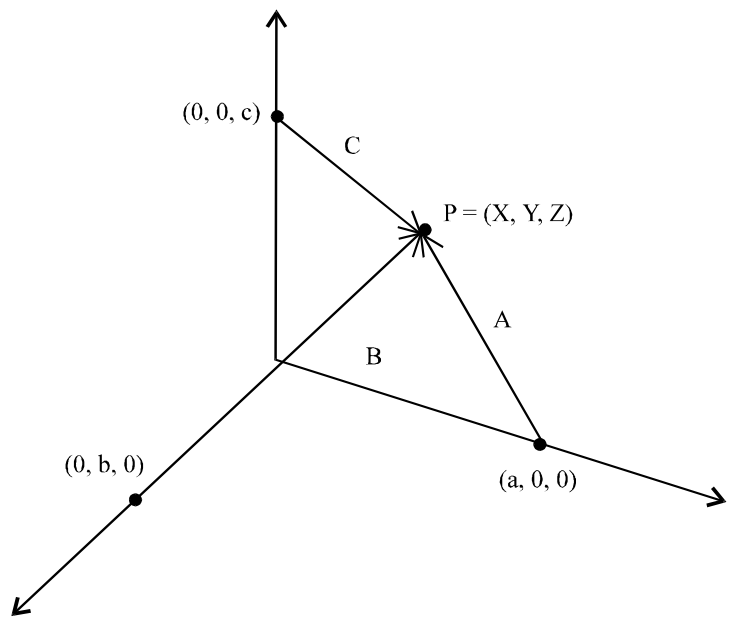

Fig. 11: Vector diagram in $3 \mathrm{D}$ space with axis $\mathrm{X}-\mathrm{Z}$
$\mathrm{S}_{1}=(\mathrm{a}, 0,0), \mathrm{S}_{3}=(0, \mathrm{~b}, 0), \mathrm{S}_{3}=(0,0, \mathrm{c})$ converging at the end point $\mathrm{P}=(\mathrm{x}, \mathrm{y}, \mathrm{z})$. Where $\mathrm{a}-\mathrm{c}$ are positive real numbers. The magnitude of A-C are:

$$
\begin{aligned}
& |A|=\sqrt{\left((x-a)^{2}+(y-0)^{2}+(z-0)^{2}\right)} \\
& |B|=\sqrt{\left((x-0)^{2}+(y-b)^{2}+(z-0)^{2}\right)} \\
& |C|=\sqrt{\left((x-0)^{2}+(y-0)^{2}+(z-0)^{2}\right)}
\end{aligned}
$$

Squaring both sides and expanding the brackets we have:

$$
\begin{aligned}
& |A|=x^{2}-2 x a+a^{2}+y^{2}+z^{2} \\
& |B|=y^{2}-2 y b+b^{2}+x^{2}+z^{2} \\
& |C|=z^{2}-2 z c+c^{2}+x^{2}+y^{2}
\end{aligned}
$$

Since, a-c, $|\mathrm{A}|,|\mathrm{B}|,|\mathrm{C}|$ are known values, the system of simultaneous equations can be solved to obtain the variables in the co-ordinates $(\mathrm{x}-\mathrm{z})$ of the convergence point $P$.

This method estimates the position of the object by calculating the converging point of vectors with magnitudes equated to the distance measurements. This method is applied in a function in the operational control code of the system to be called intermittently to obtain the approximate position of the object, thus, tracking the motion in $3 \mathrm{D}$.

Overview of implementation limitations: Listed below are some major difficulties and challenges experienced during the implementation of this system.

Limited sensing range: The ultrasonic sensor is selected for a few reasons, one important one being its range $(4 \mathrm{~m}$ maximum) and accuracy, in the real world implementations, a higher range will likely be needed to cover larger regions of space.

A solution option is to select an ultrasonic sensor with higher range (note this will increase cost). Another option is to place the sensors in a mesh like formation (requires an increase in the number of sensors).

Large firing sequence interval: The interval between pulse triggers between sensors in the firing sequence is large due to the necessity for each sensor to wait for its 
echo before the next sensor is triggered. The larger the sensing interval, the lower the number of measurements taken per any unit time, thus, reducing the tracking accuracy increasingly with faster moving objects.

To solve this problem, the sensor settings can be adjusted by giving each sensor a different pulse frequency, so that, each sensor listens for a different echo. This way pulses may be firing simultaneously without fear of interference.

Identification of more than one body/object: When more than one object is within the sensing space the system has no way of reading this situation because it listens for only one echo, therefore, the first echo received will be used to determine distance. A way to solve this is to set the sensors to listen to and record multiple echoes within the space. The code and the functional algorithms can be adjusted to decipher which measurement are associated to each sensors

Summary: This study examined the use of electronic technology to sense and track the motion of bodies in a region of 3 dimensional space. Due to the fact that people cannot be everywhere at once, it is reasonable that technology can be a source for a solution in which more space can be surveilled without the presence of a person at that moment.

\section{CONCLUSION}

This study showed that the motion sensing and tracking device implemented with the use of ultrasonic sensors can accurately and effectively monitor and track objects in a 3 dimensional space. It is an important tool in physical security and an integral part of security systems. Its usefulness in the field of security whether it be in domestic use for home based monitoring, workplace and industry surveillance cannot be overstated. There are also possible military applications. Finally in athletics, it can be used to acquire accurate performance reviews of athletes ranging from variations in movement to the change of pace of athletes.

\section{RECOMMENDATION}

The technology employed in this study has a wide range of applications which should be explored. One of such more advance application of ultrasonic sensors is in the 3D mapping of object shapes and topography.

\section{ACKNOWLEDGEMENT}

This research was done with the help of current and previous students of wireless communication and sensors
Research Group of the Computer Science Department of the School of Computing and Engineering Sciences, Babcock University Ilishan-Remo, Nigeria and finally, our special thanks to Dr Adesegun Olusegun for his valuable comments and suggestions to improve this study.

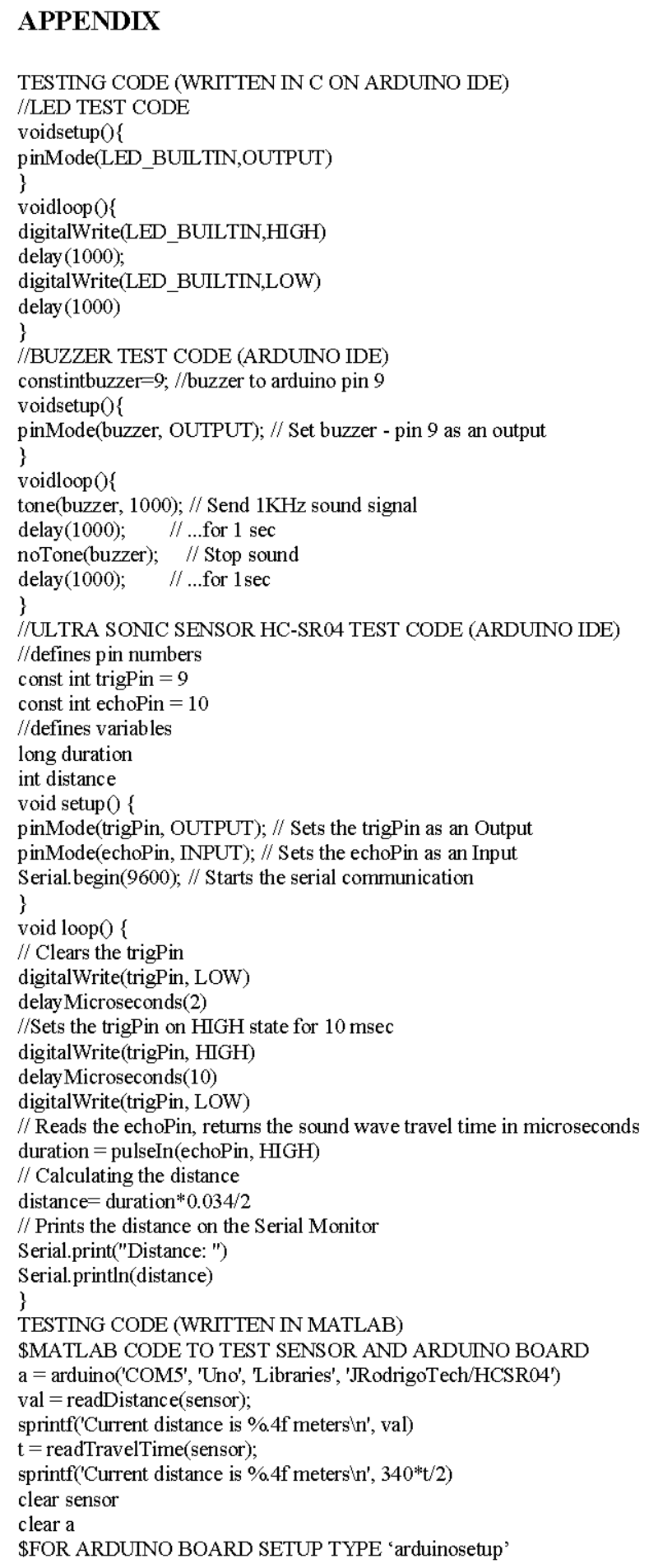




\section{REFERENCES}

Agarwal, T., 2017. Different types of motion sensors, working and application. EDGEFX.ПI Kits \& Solution, Mumbai, India. https://www.edgefx.in/types-ofmotion-sensors-working-and-applications/

Anonymous, 2019a. How do motion sensors work? A beginners guide by SafeWise. SafeWise.com, USA. https://www.pinterest.com/pin/ 235313149255825799/

Anonymous, 2019b. How radar detectors work. Thomas Publishing Company, New York, USA. https://www. thomasnet.com/articles/instruments-c ontrols/how-radar-detectors-work

Anonymous, 2019c. Infrared sensors. Atlassian, Sydney, Australia. https://wiki.metropolia.fi/display/sensor /Infrared+sensors

Anonymous, 2019d. What is a motion sensor alarm?. Kisi Inc., New York, USA. https://www.getkisi.com /guides/motion-sensor-alarm
Li, C., J. Cummings, J. Lam, E. Graves and W. Wu, 2009. Radar remote monitoring of vital signs. IEEE. Microwave Mag., 10: 47-56.

Mohammadzadeh, F.F., S. Liu, K.A. Bond and C.S. Nam, 2015. Feasibility of a wearable, sensor-based motion tracking system. Proceedings of the 6 th International Conference and Affiliated Conferences on Applied Human Factors and Ergonomics (AHFE 2015) Vol. 03, July 26-30, 2015, Elsevier Publishing Company, Las Vegas, Nevada, USA., pp: 192-199.

Sun, T., H. Li, Q. Liu, L. Duan and M. Li et al., 2017. Inertial sensor-based motion analysis of lower limbs for rehabilitation treatments. J. Healthcare Eng., 2017: $1-11$.

Xu, C., J. He, X. Zhang, X. Zhou and S. Duan, 2019. Towards human motion tracking: Multi-sensory IMU/TOA fusion method and fundamental limits. Electron., 8: 1-22.

Zungeru, A.M., 2013. Design and development of an ultrasonic motion detector. Intl. J. Secur. Privacy Trust Manage., 2: 1-13. 